\title{
SCATTERING CHARACTERISTICS OF 3-D DISCONTINUITY CONSISTING OF SEMICONDUCTOR SAMPLE FILLED IN WAVEGUIDE WITH GAPS
}

\author{
XuShanjia, ${ }^{1}$ Wu Xinzhang, ${ }^{1}$ P. Boege, H. Schäfer, ${ }^{2}$ C. R. \\ Becker, ${ }^{2}$ and R. Geick ${ }^{2}$ \\ ${ }^{1}$ Department of Radio and Electronics \\ University of Science and Technology of China \\ Hefei, Anhui, 230026 \\ People's Republic of China \\ ${ }^{2}$ Physikalisches Institut der Universität \\ Am Hubland, 97074 Würzburg, Germany
}

Received August 6, 1993

\section{ABSTRACT}

The scattering characteristics of 3-D discontinuity consisting of II-VI-epitaxial layer grown on lossy dielectric substrate inserted in the waveguide with gaps are investigated in detail by the method which combines the multimode net work theory with the rigorous mode matching procedure. The experimental data verify the accuracy and the effectiveness of the present method.

\section{INTRODUCTION}

Recently, II-VI semiconductors have become more and more important in material science and engineering because of their inherent advantages for applications in optoelectronic, infrared and millimeter wave techniques. For these purposes the exact knowledge of their electric properties, e.g. conductivity, are of essential significance. However, poorly conducting II-VI semicondutors have always created 
problems. In many cases, it is extremely difficulty or even impossible to obtain good ohmic contacts. For characterising II-VI compounds under these circumstances, a contactless conductivity measurement [1] has been developped employing a microwave bridge technique. In this method, the determination of property parameters of a semiconductor is transfered to the measurement of the scattering characteristics of the corresponding samples. The key point of realizing this idea is to theoretically determine the relationship between the property parameters of the semiconductor and scattering parameters of the corresponding samples. In the former measurement, the height of the semiconductor sample was chosen to be the same as the waveguide so that the theoretical analysis for the boundary value problem became easier; because in that case it is a two dimensional scattering problem as previously have done using the multireflection method[1], the multimode network method[2] and the high-order finite element method[3]. However, in that case, special care must be taken in the measurement to ensure good electric contact between the sample and the waveguide walls[1]. It is inconvenient and time consuming. Besides, to satisfy the particular dimension requirement, the height dimension of the sample has to be made in a very precise way; it is not so convenient and in some cases is rather difficult.

In order to avoid above mentioned disadvantages and to make the contactless microwave measurement a simple routine to determine the properties of the semiconductor with sufficient accuracy in a sufficiently short time, in this paper we propose a new sample mounting structure as shown in Fig.1. The sample having different height with the waveguide, includes an epitaxial layer $\left(\epsilon_{p}\right)$ and a lossy dielectric slab $\left(\epsilon_{s}\right)$ as substrate. Two dielectric films having the dielectric constant $\epsilon_{f}$ and $\epsilon_{b}$ about 2.0 are introduced as two transient regions to get good microwave conducting between the sample and the walls of the waveguide. The other two dielectric blocks having the dielectric constants around 1.0 together with the films form the mounting structure to precisely fix the position of the sample in the waveguide so that the repeatability of the measurement is guaranteed. Although the new mounting structure really makes the measurement much quicker and more reliable, the improvements require an extended and more elaborate mathematical analysis of the experimental data, as a result, 
the theoretical analysis becomes much more complicated as compared to the simple situation of the partially filled waveguide before. Thus in the present case it is a complicated three dimensional scattering problem. The following two aspects make the problem even more difficult. First, the conductivity of the epitaxial layer may vary in a wide range from ten to some thousands $(\mathrm{mhos} / \mathrm{cm})$ to satisfy the practical requirements for different devices. As a consequence, the real and imaginary part of the corresponding complex dielectric constants may be very large, especially the imaginary part may vary from several hundreds to some millions. If the calculation is not carried out properly, it may suffer from convergence problem. In addition, because the epitaxial layer is neither an ideal dielectric nor ideal conductor, some assumptions for these two extreme circumstances can not be used, for instance, one can not simplify the problem with approximate methods such as perturbation theory. Secondly, the epitaxial layer is very thin, only 1 to $5 \mu \mathrm{m}$, or even less than $1 \mu \mathrm{m}$, but it can not be assumed to be zero, because investigating the effect of the thickness on scattering characteristics is one of our tasks. Therefore, it may also cause a convergence problem if the method is not correctly adopted in analyzing the present discontinuity which creates the current distribution function with high order singularities.

In this paper, an accurate and relatively simple method is used to analyze the scattering characteristics of 3-D discontinuity consisting of the II-VI semiconductor sample partially filled in waveguide with two dielectric gaps. Since the present method combines the multimode network theory with the rigorous mode matching procedure and transfers the electromagnetic field boundary value problem into a multimode network analysis problem, the whole calculation procedure is simplified while still retaining the high accuracy of the mode matching method.

The validity of the present approach is justified by comparisons of the transmission coefficients for different samples with different gaps between the experimental data and theoretical predictions. 


\section{METHOD OF ANALYSIS}

Fig. 1 shows the cross section of the structure under consideration. This discontinuity structure is corresponding to a semiconductor sample partially filled in the waveguide with film gaps. As described in the last section, the sample having different height than the waveguide consists of an epitaxial layer and a lossy dielectric slab as substrate. Two dielectric films are introduced as the transient regions to ensure the good microwave conducting between the sample and the waveguide; the other two dielectric blocks together with the films form the mounting structure to precisely fix the position of the sample in the waveguide. We would like to calculate the scattering characteristics of this 3-D discontinuity problem. The solution procedure of the problem may be divided into two steps. First, analyze the eigenvalue problem of the two waveguides respectively in the transverse cross section; secondly, calculate the scattering characteristics of the discontinuity in the longitudinal direction, in which the symmetrical property of the structure in the longitudinal direction is used to simplify the calculation procedure.
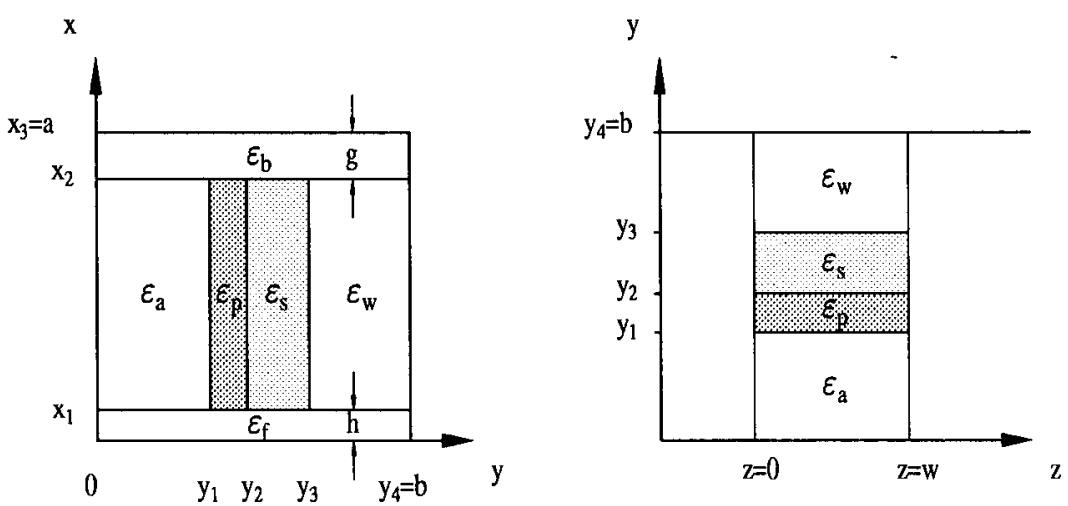

Fig.1 Cross section of the stratified lossy dielectric blocks in the rectangular waveguide. 


\section{STEP I. Analysis of the Eigenvalue Problem for the Two Waveguides}

\section{Eigenvalue problem of the empty waveguide}

It is well known that the eigenvalues and eigenfunctions for the empty rectangular waveguide are respectively as shown in Table 1. Here we use a single prime to denote quantities for $\mathrm{H}$ modes, a double prime for $\mathrm{E}$ modes and no prime for either $\mathrm{H}$ modes or $\mathrm{E}$ modes. It should be emphasized that in order to accelerate the convergence in the mode matching calculation, the sequence of the $\mathrm{H}$ and $\mathrm{E}$ modes must be rearranged according to the eigenvalues $k_{c}$ from small to large.

Table 1. Eigenfunctions and field components of the $H$ and $E$ modes in the empty rectangular waveguide.

$$
\begin{aligned}
& \mathrm{H} \text { mode } \\
& e_{x}^{\prime}=A^{\prime} q_{y}^{\prime} \cos q_{x}^{\prime} x \sin q_{y}^{\prime} y \\
& \text { E mode } \\
& e_{y}^{\prime}=-A^{\prime} q_{x}^{\prime} \sin q_{x}^{\prime} x \cos q_{y}^{\prime} y \\
& e_{x}^{\prime \prime}=A^{\prime \prime} q_{x}^{\prime \prime} \cos q_{x}^{\prime \prime} x \sin q_{y}^{\prime \prime} y \\
& h_{x}^{\prime}=A^{\prime} q_{x}^{\prime} \sin q_{x}^{\prime} x \cos q_{y}^{\prime} y \\
& \epsilon_{y}^{\prime \prime}=A^{\prime \prime} q_{y}^{\prime \prime} \sin q_{x}^{\prime \prime} x \cos q_{y}^{\prime \prime} y \\
& h_{y}^{\prime}=A^{\prime} q_{y}^{\prime} \cos q_{x}^{\prime} x \sin q_{y}^{\prime} y \\
& h_{x}^{\prime \prime}=-A^{\prime \prime} q_{y}^{\prime \prime} \sin q_{x}^{\prime \prime} x \cos q_{y}^{\prime \prime} y \\
& E_{x}^{\prime}=U^{\prime}(z) e_{x}^{\prime}(x, y) \\
& h_{y}^{\prime \prime}=A^{\prime \prime} q_{x}^{\prime \prime} \cos q_{x}^{\prime \prime} x \sin q_{y}^{\prime \prime} y \\
& E_{y}^{\prime}=U^{\prime}(z) e_{y}^{\prime}(x, y) \\
& E_{x}^{\prime \prime}=U^{\prime \prime}(z) e_{x}^{\prime \prime}(x, y) \\
& E_{z}^{\prime}=0 \\
& E_{y}^{\prime \prime}=U^{\prime \prime}(z) e_{y}^{\prime \prime}(x, y) \\
& H_{x}^{\prime}=J^{\prime}(z) h_{x}^{\prime}(x, y) \\
& E_{z}^{\prime \prime}=J^{\prime \prime}(z) B^{\prime \prime} \sin q_{x}^{\prime \prime} x \sin q_{y}^{\prime \prime} y \\
& H_{y}^{\prime}=J^{\prime}(z) h_{y}^{\prime}(x, y) \\
& H_{x}^{\prime \prime}=J^{\prime \prime}(z) h_{x}^{\prime \prime}(x, y) \\
& H_{z}^{\prime}=U^{\prime}(z) B^{\prime} \cos q_{x}^{\prime} x \cos q_{y}^{\prime} y \quad H_{z}^{\prime \prime}=0 \\
& A^{\prime}=C_{m n} \sqrt{\gamma_{m} \gamma_{n}} / \pi \\
& A^{\prime \prime}=-2 C_{m n} / \pi \\
& B^{\prime}=-j \sqrt{\frac{t_{0}}{\varepsilon_{0}}} \lambda \sqrt{\gamma_{m} \gamma_{n}} C_{m n} \\
& B^{\prime \prime}=-j \sqrt{\frac{\mu_{0}}{\varepsilon_{0}}} \frac{\lambda \sqrt{\gamma_{m} \gamma_{n}}}{a b} C_{m n} \\
& C_{m n}=1 / \sqrt{m^{2}(b / a)+n^{2}(a / b)} \\
& \left.\gamma_{m, n}=1 \quad \text { (if } m \text { or } n=0\right) \\
& \left.\gamma_{m, n}=2 \quad \text { (if } m \text { and } n \neq 0\right), \quad(m=n=0 \text { excluded) } \\
& q_{x}=m \pi / a, \quad q_{y}=n \pi / b, \quad q_{c}^{2}=q_{x}^{2}+q_{y}^{2}
\end{aligned}
$$


In Table $1, U^{\prime}(z), U^{\prime \prime}(z), J^{\prime}(z)$ and $J^{\prime \prime}(z)$ are respectively, the voltage and current amplitudes of the $\mathrm{H}$ and $\mathrm{E}$ eigenmodes; they satisfy the transmission line equation:

$$
\begin{array}{ll}
\frac{d U^{\prime}(z)}{d z}=-j q_{z}^{\prime} Z_{c}^{\prime} J^{\prime}(z), & \frac{d J^{\prime}(z)}{d z}=-j q_{z}^{\prime} Y_{c}^{\prime} U^{\prime}(z) \\
\frac{d U^{\prime \prime}(z)}{d z}=-j q_{z}^{\prime \prime} Z_{c}^{\prime \prime} J^{\prime \prime}(z), & \frac{d J^{\prime \prime}(z)}{d z}=-j q_{z}^{\prime \prime} Y_{c}^{\prime \prime} U^{\prime \prime}(z) .
\end{array}
$$

where the propagation wavenumber $q_{z}$, the characteristic impedance and admittance $Z_{c}^{\prime}, Z_{c}^{\prime \prime}, Y_{c}^{\prime}$ and $Y_{c}^{\prime \prime}$ are given by:

$$
\begin{gathered}
q_{z}^{2}=q_{0}^{2}-q_{c}^{2} \\
Z_{c}^{\prime}=\frac{1}{Y_{c}^{\prime}}=\frac{\omega \mu_{0}}{q_{z}^{\prime}}, Z_{c}^{\prime \prime}=\frac{1}{Y_{c}^{\prime \prime}}=\frac{q_{z}^{\prime \prime}}{\omega \epsilon_{0}} .
\end{gathered}
$$

The mode functions $\mathbf{e}^{\prime}, \mathbf{h}^{\prime}, \mathbf{e}^{\prime \prime}$, and $\mathbf{h}^{\prime \prime}$ possess the vector orthogonality properties

$$
\begin{aligned}
& \iint\left(\mathbf{e}_{i}^{\prime} \times \mathbf{h}_{j}^{\prime}\right) \cdot \mathbf{d} \mathbf{s}=\delta_{i j}, \quad \iint\left(\mathbf{e}_{i}^{\prime \prime} \times \mathbf{h}^{\prime \prime}{ }_{j}\right) \cdot \mathbf{d} \mathbf{s}=\delta_{i j} \\
& \iint\left(\mathbf{e}_{i}^{\prime} \times \mathbf{h}_{j}^{\prime \prime}\right) \cdot \mathbf{d} \mathbf{s}=0, \quad \iint\left(\mathbf{e}_{i}^{\prime \prime} \times \mathbf{h}_{j}^{\prime}\right) \cdot \mathbf{d} \mathbf{s}=0
\end{aligned}
$$

where $i$ and $j$ represent a pair of mode indices $m, n$, respectively. The transverse electric and magnetic fields in the waveguide can be expressed in terms of the above defined orthogonal functions by means of the representation:

$$
\begin{aligned}
& \mathbf{E}_{\mathbf{t}}(x, y, z)=\sum_{i} U_{i}^{\prime}(z) \mathbf{e}_{i}{ }_{i}(x, y)+\sum_{i} U_{i}^{\prime \prime}(z) \mathbf{e}^{\prime \prime}{ }_{i}(x, y), \\
& \mathbf{H}_{\mathbf{t}}(x, y, z)=\sum_{i} J_{i}^{\prime}(z) \mathbf{h}_{i}^{\prime}(x, y)+\sum_{i} J_{i}^{\prime \prime}(z) \mathbf{h}^{\prime \prime}{ }_{i}(x, y) .
\end{aligned}
$$




\section{Eigenvalue problem of the lossy dielectric blocks (sample) filled waveguide}

Fig.2 shows the discontinuity structure and its equivalent multimode network representation in the transverse cross section, there are three $\left(\Delta_{l}, l=1,2,3\right)$ constituent parts. Each constituent supports two kinds of eigenmodes; one is the LSE mode ( $H^{(y)}$ type modes, characterized by $E_{y}=0$ ) and the other is the LSM modes ( $E^{(y)}$ type modes, characterized by $H_{y}=0$ ). Table 2 gives the eigenfunctions and field components of the eigenmode in each multilayer planar dielectric structure.

Table 2. Eigenfunctions and field components of the eigenmode in each multilayer planar dielectric structure

LSE or $\mathrm{H}^{(y)}$ mode

$e_{x}^{\prime}=\left(k_{0}^{2} / k_{u}^{\prime 2}\right) I^{\prime}(x) \phi^{\prime}(y)$

$e_{y}^{\prime}=0$

$h_{x}^{\prime}=\left[-j /\left(\omega \mu_{0}\right)\right] V^{\prime}(x) \dot{\phi}^{\prime}(y)$

$h_{y}^{\prime}=I^{\prime}(x) \phi^{\prime}(y)$

$E_{x}^{\prime}=e_{x}^{\prime}(x, y) U(z)$

$E_{y}^{\prime}=0$

$E_{z}^{\prime}=-V^{\prime}(x) \phi^{\prime}(y) J(z)$

$H_{x}^{\prime}=h_{x}^{\prime}(x, y) J(z)$

$H_{y}^{\prime}=h_{y}^{\prime}(x, y) J(z)$

$H_{z}^{\prime}=C^{\prime} I^{\prime}(x) \dot{\phi}^{\prime}(y) U(z)$

$C^{\prime}=-j \omega \epsilon_{0} / k_{u}^{2}$
LSM or $\mathrm{E}^{(y)}$ mode

$e_{x}^{\prime \prime}=\left[-1 /\left(k_{z} \epsilon(y)\right)\right] I^{\prime \prime}(x) \dot{\phi}^{\prime \prime}(y)$

$e_{y}^{\prime \prime}=\left[-j \omega \epsilon_{0} /\left(k_{z} \epsilon(y)\right)\right] V^{\prime \prime}(x) \phi^{\prime \prime}(y)$

$h_{x}^{\prime \prime}=\left(j \omega \epsilon_{0} k_{z} / k_{u}^{\prime 2}\right) V^{\prime \prime}(x) \phi^{\prime \prime}(y)$

$h_{y}^{\prime \prime}=0$

$E_{x}^{\prime \prime}=e_{x}^{\prime \prime}(x, y) U(z)$

$E_{y}^{\prime \prime}=e_{y}^{\prime \prime}(x, y) U(z)$

$E_{z}^{\prime \prime}=\left[k_{z} /\left(k_{u}^{\prime \prime 2} \epsilon(y)\right)\right] V^{\prime \prime}(x) \dot{\phi}^{\prime \prime}(y) J(z)$

$H_{x}^{\prime \prime}=h_{x}^{\prime \prime}(x, y) J(z)$

$H_{y}^{\prime \prime}=0$

$H_{z}^{\prime \prime}=C^{\prime \prime} I^{\prime \prime}(x) \phi^{\prime \prime}(y) U(z)$

$C^{\prime \prime}=-j \omega \epsilon_{0} / k_{z}$ 

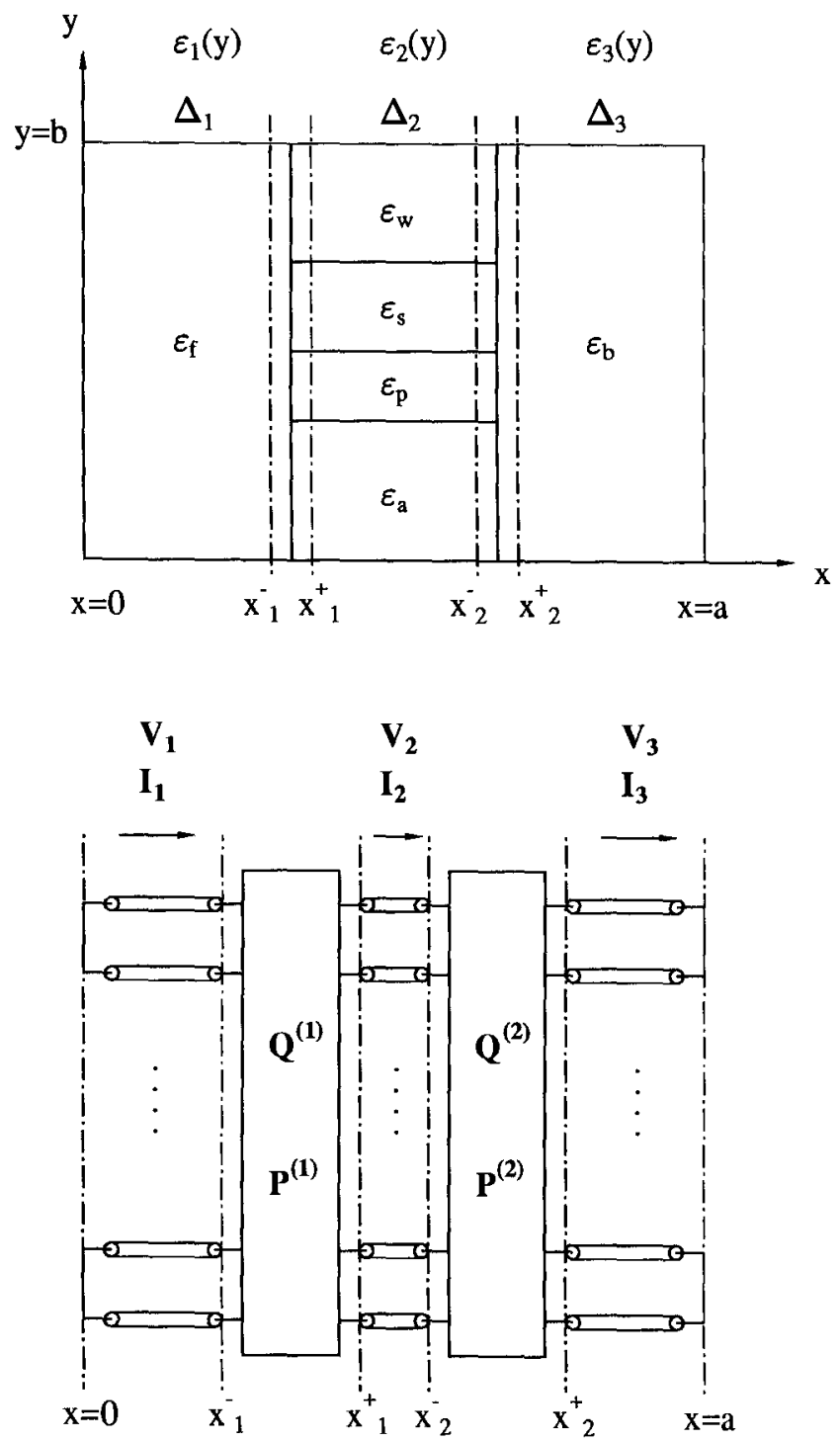

Fig.2 Discontinuity structure in transverse cross section and its equivalent network representation. 
In Table 2, we use a single prime to denote quantities for the LSE modes and a double prime for the LSM modes. $\dot{\phi}(y)$ denotes the derivative of $\phi(y)$ with respect to y. $U(z)$ and $J(z)$ are respectively the voltage and current amplitudes of the eigenmode in the sample filled waveguide, they satisfy the following transmission line equation:

$$
\begin{aligned}
& \frac{d U(z)}{d z}=-j k_{z} Z_{c} J(z), \\
& \frac{d J(z)}{d z}=-j k_{z} Y_{c} U(z) ;
\end{aligned}
$$

where $k_{z}$ is the longitudinal propagation constant of the eigenmode in the sample filled waveguide and $Z_{c}$ is the characteristic impedance of the equivalent transmission line in the $\mathrm{z}$ direction, which is defined by

$$
Z_{c}=\frac{1}{Y_{c}}=\frac{k_{z}}{\omega \epsilon_{0}} \text {. }
$$

It is noted that the boundary conditions at the discontinuity require the total tangential field components be continuous across the discontinuity, and a necessary condition for the continuity of the tangential field components is that every mode in the constituent regions must have the same propagation wavenumber $k_{z}$, in the direction along the discontinuity. Equation (14) reflects this fact.

In Table 2, $V^{\prime}(x), V^{\prime \prime}(x), I^{\prime}(x)$ and $I^{\prime \prime}(x)$ can be interpreted as the voltage and current respectively for the LSE and LSM modes; they satisfy the transmission line equations

$$
\begin{array}{cc}
\frac{d V^{\prime}(x)}{d x}=-j k_{x}^{\prime} Z_{o}^{\prime} I^{\prime}(x) & \frac{d I^{\prime}(x)}{d x}=-j k_{x}^{\prime} Y_{o}^{\prime} V^{\prime}(x) \\
\frac{d V^{\prime \prime}(x)}{d x}=-j k_{x}^{\prime \prime} Z_{o}^{\prime \prime} I^{\prime \prime}(x) & \frac{d I^{\prime \prime}(x)}{d x}=-j k_{x}^{\prime \prime} Y_{o}^{\prime \prime} V^{\prime \prime}(x) \\
k_{x}^{\prime 2}+k_{z}^{2}=k_{u}^{\prime 2} & k_{x}^{\prime 2}+k_{z}^{2}=k_{u}^{\prime 2} \\
Z_{o}^{\prime}=\frac{1}{Y_{o}^{\prime}}=\frac{\omega \mu_{0} k_{x}^{\prime}}{k_{u}^{\prime 2}}=\frac{k_{x}^{\prime}}{\omega \epsilon_{0} \epsilon_{e}^{\prime}}
\end{array}
$$




$$
\begin{aligned}
& Z_{o}^{\prime \prime}=\frac{1}{Y_{o}^{\prime \prime}}=\frac{k_{u}^{\prime \prime 2}}{\omega \epsilon_{0} k_{x}^{\prime \prime}}=\frac{\omega \mu_{0} \epsilon_{e}^{\prime \prime}}{k_{x}^{\prime \prime}} ; \\
& \epsilon_{e f f}^{\prime}=\frac{k_{u}^{\prime 2}}{k_{0}^{2}}, \quad \epsilon_{e f f}^{\prime \prime}=\frac{k_{u}^{\prime 2}}{k_{0}^{2}} .
\end{aligned}
$$

From Table 2 it is found that once the eigenvalues $k_{u}^{\prime}, k_{u}^{\prime \prime}$, and the eigenfunctions $V^{\prime}(x), V^{\prime \prime}(x), I^{\prime}(x), I^{\prime \prime}(x), \phi^{\prime}(y)$ and $\phi^{\prime \prime}(y)$ are obtained, the modefunctions $\mathbf{e}^{\prime}, \mathbf{e}^{\prime \prime}, \mathbf{h}^{\prime}$ and $\mathbf{h}^{\prime \prime}$ are determined. Then the modefunctions $\mathbf{e}$ and $\mathbf{h}$ for the sample filled waveguide can be expressed in terms of the superposition of the complete set of $\mathbf{e}^{\prime}, \mathbf{e}^{\prime \prime}, \mathbf{h}^{\prime}$ and $\mathbf{h}^{\prime \prime}$ as

$$
\begin{aligned}
& \mathbf{e}_{i}=\sum_{m} \mathbf{e}_{i m}^{\prime}+\sum_{n} \mathbf{e}_{i n}^{\prime \prime}, \\
& \mathbf{h}_{i}=\sum_{m} \mathbf{h}_{i m}^{\prime}+\sum_{n} \mathbf{h}_{i n}^{\prime \prime} ;
\end{aligned}
$$

and the transverse electric and magnetic fields in the sample filled waveguide can be expressed in terms of mode function $\mathbf{e}$ and $\mathbf{h}$ as

$$
\begin{aligned}
& \mathbf{E}_{t}=\sum_{i} \mathbf{e}_{i} U_{i}=\sum_{i}\left(\sum_{m} \mathbf{e}_{i m}^{\prime}+\sum_{n} \mathbf{e}^{\prime \prime}{ }_{i n}\right) U_{i}, \\
& \mathbf{H}_{t}=\sum_{i} \mathbf{h}_{i} J_{i}=\sum_{i}\left(\sum_{m} \mathbf{h}_{i m}^{\prime}+\sum_{n} \mathbf{h}^{\prime \prime}{ }_{i n}\right) J_{i} .
\end{aligned}
$$

Where $i$ denotes the mode number in the sample filled waveguide and $m, n$ the mode number in the multilayer planar dielectric structure in the constituent $\Delta_{l}$ regions. (cf. Fig. 2)

From (18)-(21) it is clear that we choose the modefunctions of multilayer dielectric waveguide as the basis functions to express the modefunctions for the sample filled waveguide. In such a way the fast convergence and less computing time can be obtained. Also, it is worth to note that each eigenmode in sample filled waveguide has six field components whereas in the empty waveguide ( $\mathrm{H}$ and $\mathrm{E}$ mode) or in every constituent guided region (LSE and LSM mode) each eigenmode has only five field components; therefore we can not separate the complete set of modefunctions into two classes ( $\mathrm{H}$ and $\mathrm{E}$ mode). It means that only one voltage and one current amplitude function 
$U_{i}, J_{i}$ are sufficient to describe the behavior of the eigenmodes in the $z$ direction. Following gives the solution procedure of the eigenvalue problem.

\section{(1) Determination of $\phi^{\prime}(y)$ and $\phi^{\prime \prime}(y)$}

Since $\phi^{\prime}(y)$ and $\phi^{\prime \prime}(y)$ are independent of variable $\mathrm{x}$, we consider each constituent region as being infinitely wide while retaining the structure unchanged in the y direction. As a result, each region becomes a four layer, or one layer infinite planar dielectric waveguide as shown in Fig.3.
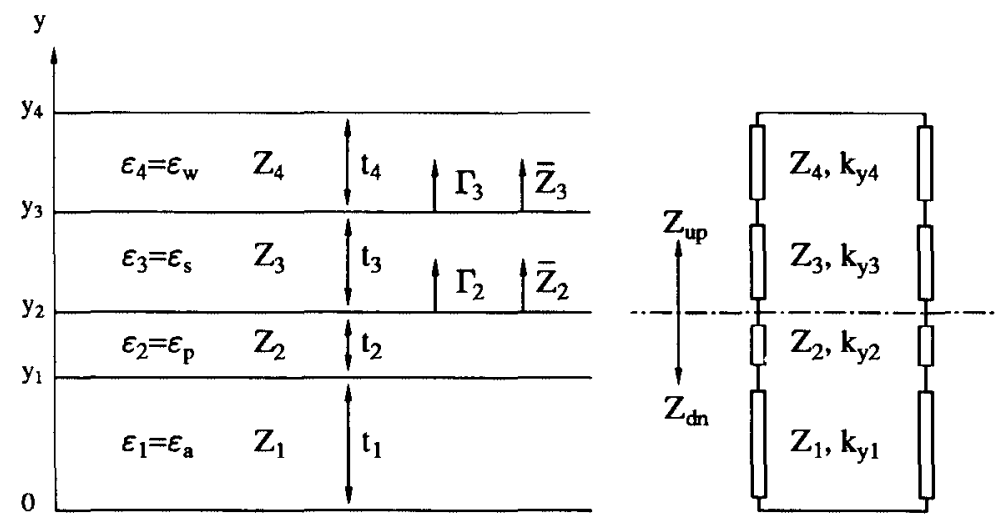

Fig.3 Multilayer planar dielectric structure and its equivalent network representation.

The solutions of $\phi^{\prime}(y)$ and $\phi^{\prime \prime}(y)$ for the four layer planar dielectric waveguide are respectively as follows:

For TE modes:

$$
\phi^{\prime}= \begin{cases}A_{1}^{\prime} \sin \left(k_{y 1} y\right) & \left(0<=y \leq y_{1}\right) \\ A_{2}^{\prime} \sin \left[k_{y 2}\left(y-y_{2}\right)-\theta_{2}\right] & \left(y_{1}<=y \leq y_{2}\right) \\ A_{3}^{\prime} \sin \left[k_{y 3}\left(y-y_{3}\right)-\theta_{3}\right] & \left(y_{2}<=y \leq y_{3}\right) \\ A_{4}^{\prime} \sin \left[k_{y 4}\left(y-y_{4}\right)\right] & \left(y_{3}<=y \leq y_{4}\right)\end{cases}
$$


For TM modes:

$$
\phi^{\prime \prime}= \begin{cases}A_{1}^{\prime \prime} \cos \left(y_{y 1} y\right) & \left(0<=y \leq y_{1}\right) \\ A_{2}^{\prime \prime} \cos \left[k_{y 2}\left(y-y_{2}\right)-\theta_{2}\right] & \left(y_{1}<=y \leq y_{2}\right) \\ A_{3}^{\prime \prime} \cos \left[k_{y 3}\left(y-y_{3}\right)-\theta_{3}\right] & \left(y_{2}<=y \leq y_{3}\right) \\ A_{4}^{\prime \prime} \cos \left[k_{y 4}\left(y-y_{4}\right)\right] & \left(y_{3}<=y \leq y_{4}\right)\end{cases}
$$

where

$$
\begin{aligned}
\theta_{2} & =j\left[\ln \left(-\Gamma_{2}\right)\right] / 2, \\
\theta_{3} & =j\left[\ln \left(-\Gamma_{3}\right)\right] / 2 ; \\
\Gamma_{2} & =\left(\bar{Z}_{2}-Z_{2}\right) /\left(\bar{Z}_{2}+Z_{2}\right), \\
\Gamma_{3} & =\left(\bar{Z}_{3}-Z_{3}\right) /\left(\bar{Z}_{3}+Z_{3}\right) ; \\
Z_{l}^{\prime} & =\omega \mu / k_{y l}^{\prime}, \\
Z_{l}^{\prime \prime} & =k_{y l}^{\prime \prime} / \omega \epsilon_{0} \epsilon_{l} ; \\
k_{y l}^{2} & =k_{0}^{2} \epsilon_{l}-k_{u}^{2}, \\
k_{u}^{2} & =k_{0}^{2} \epsilon_{c f f}, \quad l=1,2,3,4 \\
\bar{Z}_{3} & =j Z_{4} \tan \left(k_{y 4} t_{4}\right), \quad \\
\bar{Z}_{2} & =Z_{3} \frac{\bar{Z}_{3}+j Z_{3} \tan \left(k_{y 3} t_{3}\right)}{Z_{3}+j \bar{Z}_{3} \tan \left(k_{y 3} t_{3}\right)} .
\end{aligned}
$$

The amplitudes $A_{i}$ can be determined with the boundary condi tions at each interface as follows:

For TE modes:

$$
\begin{aligned}
& A_{2}^{\prime}=-A_{1}^{\prime} \sin \left(k_{y 1} t_{1}\right) / \sin \left(k_{y 2} t_{2}+\theta_{2}\right) \\
& A_{3}^{\prime}=A_{2}^{\prime} \sin \theta_{2} / \sin \left(k_{y 3} t_{3}+\theta_{3}\right) \\
& A_{4}^{\prime}=A_{3}^{\prime} \sin \theta_{3} / \sin \left(k_{y 4} t_{4}\right)
\end{aligned}
$$


For TM modes:

$$
\begin{aligned}
& A_{2}^{\prime \prime}=A_{1}^{\prime \prime} \cos \left(k_{y 1} t_{1}\right) / \cos \left(k_{y 2} t_{2}+\theta_{2}\right) \\
& A_{3}^{\prime \prime}=A_{2}^{\prime \prime} \cos \theta_{2} / \cos \left(k_{y 3} t_{3}+\theta_{3}\right) \\
& A_{4}^{\prime \prime}=A_{3 n}^{\prime \prime} \cos \theta_{3} / \cos \left(k_{y 4} t_{4}\right)
\end{aligned}
$$

It has been proved that the eigenfunctions $\phi^{\prime}(y)$ and $\phi^{\prime \prime}(y)$ for different modes possess the following orthonormality relation

$$
\begin{gathered}
<\phi_{m}^{\prime}(y) \mid \phi_{n}^{\prime}(y)>=\int_{0}^{b} \phi_{m}^{\prime}(y) \phi_{n}^{\prime}(y) d y=\delta_{m n}, \\
<\phi_{m}^{\prime \prime}(y)\left|\frac{1}{\epsilon(y)}\right| \phi_{n}^{\prime \prime}(y)>=\int_{0}^{b} \phi_{m}^{\prime \prime}(y) \frac{1}{\epsilon(y)} \phi_{n}^{\prime \prime}(y) d y=\delta_{m n}, \\
\left(k_{u m}^{\prime}\right)^{2}<\phi_{m}^{\prime}(y)\left|\frac{1}{\epsilon(y)}\right| \dot{\phi}_{n}^{\prime \prime}(y)>+ \\
\left(k_{u n}^{\prime \prime}\right)^{2}<\phi_{n}^{\prime \prime}(y)\left|\frac{1}{\epsilon(y)}\right| \dot{\phi}_{m}^{\prime}(y)>=0 \\
\left(k_{u m}^{\prime}\right)^{2} \int_{0}^{b} \phi_{m}^{\prime}(y) \frac{1}{\epsilon(y)} \dot{\phi}_{n}^{\prime \prime}(y) d y+ \\
\left(k_{u n}^{\prime \prime}\right)^{2} \int_{0}^{b} \phi_{n}^{\prime \prime}(y) \frac{1}{\epsilon(y)} \dot{\phi}_{m}^{\prime}(y) d y=0 .
\end{gathered}
$$

The amplitude $A_{1}^{\prime}$ and $A_{1}^{\prime \prime}$, then all the normalized amplitudes of the eigenfunctions can be obtained from the orthonormal relation:

$$
\int_{0}^{b} \phi_{m}^{\prime 2} d y=1, \quad \int_{0}^{b} \phi_{m}^{\prime 2} \frac{1}{\epsilon(y)} d y=1
$$


The eigenvalues $k_{u m}^{\prime}$ and $k_{u n}^{\prime \prime}$ are calculated with the transverse resonance method as

$$
Z_{u p}+Z_{d n}=0
$$

at any reference plane in principle. However, because the conductivity of the epitaxial layer is very large in the present case, it is better to choose the upper or the lower interface of the epitaxial layer as a reference plane to avoid missing modes.

(2) Determination of $k_{z}, I(x)$ and $V(x)$

It can be seen from Fig. 2 that there are three discontinuities in the cross section. In order to get the general idea of the method and to make the explanation more convenient we had better consider the lth discontinuity first, which is shown in Fig.4 together with its equivalent network representation.

Referring to Fig.4, we observe that the tangential components of the fields at the l-th discontinuity consist of the $y$ and $z$ components and we shall therefore consider only those components explicitly. As stated earlier, the general field solution in each constituent region may be expressed in terms of the superposition of the complete set of mode functions. For the tangential field components in the $\Delta_{l}$ region $\left(x_{l-1} \leq x \leq x_{l}\right)$, from Table 2, we have

$$
\begin{aligned}
& E_{y}(x, y, z)=-j \sum_{n=1}^{\infty} V_{n}^{\prime \prime}(x) \phi_{n}^{\prime \prime}(y) \frac{\omega \epsilon_{0}}{\epsilon(y) k_{z}} U(z) \\
& E_{z}(x, y, z)=-\left[\sum_{n=1}^{\infty} V_{n}^{\prime}(x) \phi_{n}^{\prime}(y)+\sum_{n=1}^{\infty} V_{n}^{\prime \prime}(x) \psi_{n}^{\prime \prime}(y)\right] J(z) \\
& H_{y}(x, y, z)=\sum_{n=1}^{\infty} I_{n}^{\prime}(x) J(z) \phi_{n}^{\prime}(y) \\
& H_{z}(x, y, z)=-j\left[\sum_{n=1}^{\infty} I_{n}^{\prime}(x) \psi_{n}^{\prime}(y)+\sum_{n=1}^{\infty} I_{n}^{\prime \prime}(x) \phi_{n}^{\prime \prime}(y)\right] U(z)
\end{aligned}
$$



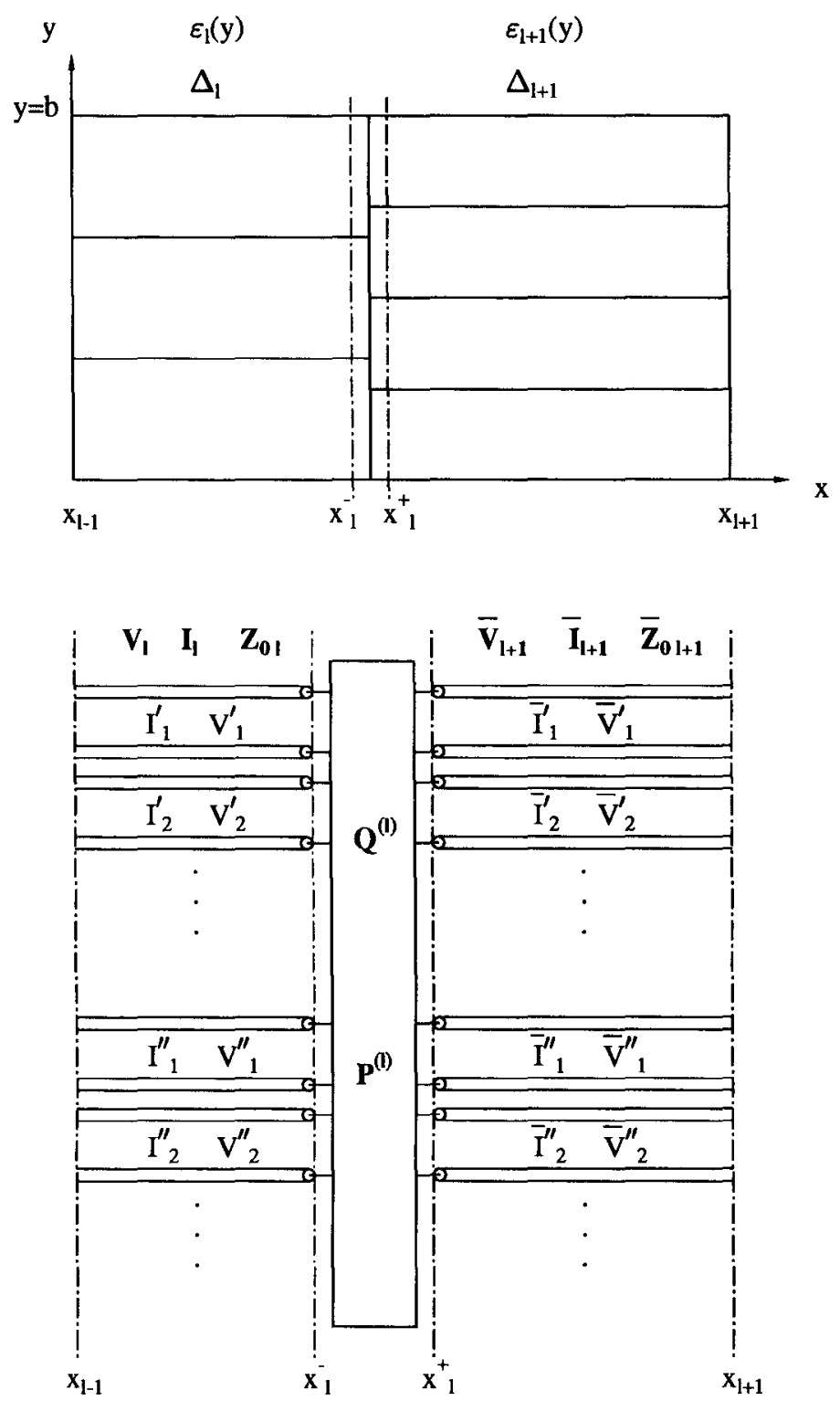

Fig.4 The 1-th discontinuity structure and its equivalent network representation. 
where we employ simplified notations

$$
\begin{aligned}
\psi_{n}^{\prime}(y) & =\frac{k_{z}}{k_{u n}^{2}} \frac{d}{d y} \phi_{n}^{\prime}(y), \\
\psi_{n}^{\prime \prime}(y) & =\frac{k_{z}}{k_{u n}^{\prime 2}} \frac{1}{\epsilon(y)} \frac{d}{d y} \phi_{n}^{\prime \prime}(y) .
\end{aligned}
$$

A similar set of tangential field components may also be written for the $x_{l} \leq x \leq x_{l+1}$ region, but it is omitted here for simplicity.

At the step discontinuity, at $x=x_{l}$, the tangential field components must be continuous. From (42)-(45), we obtain

$$
\begin{aligned}
& \sum_{n=1}^{\infty} V_{n}^{\prime \prime}\left(x_{l}\right) \phi_{n}^{\prime \prime}(y) \frac{1}{\epsilon(y)}=\sum_{n=1}^{\infty} \bar{V}_{n}^{\prime \prime}\left(x_{l}\right) \bar{\phi}_{n}^{\prime \prime}(y) \frac{1}{\bar{\epsilon}(y)}, \\
& \sum_{n=1}^{\infty} V_{n}^{\prime}\left(x_{l}\right) \phi_{n}^{\prime}(y)+\sum_{n=1}^{\infty} V_{n}^{\prime \prime}\left(x_{l}\right) \psi_{n}^{\prime \prime}(y)= \\
& \sum_{n=1}^{\infty} \bar{V}_{n}^{\prime}\left(x_{l}\right) \bar{\phi}_{n}^{\prime}(y)+\sum_{n=1}^{\infty} \bar{V}_{n}^{\prime \prime}\left(x_{l}\right) \bar{\psi}_{n}^{\prime \prime}(y), \\
& \sum_{n=1}^{\infty} I_{n}^{\prime}\left(x_{l}\right) \phi_{n}^{\prime}(y)=\sum_{n=1}^{\infty} \bar{I}_{n}^{\prime}\left(x_{l}\right) \bar{\phi}_{n}^{\prime}(y), \\
& \sum_{n=1}^{\infty} I_{n}^{\prime}\left(x_{l}\right) \psi_{n}^{\prime}(y)+\sum_{n=1}^{\infty} I_{n}^{\prime \prime}\left(x_{l}\right) \phi_{n}^{\prime \prime}(y)= \\
& \sum_{n=1}^{\infty} \bar{I}_{n}^{\prime}\left(x_{l}\right) \bar{\psi}_{n}^{\prime}(y)+\sum_{n=1}^{\infty} \bar{I}_{n}^{\prime \prime}\left(x_{l}\right) \bar{\phi}_{n}^{\prime \prime}(y) .
\end{aligned}
$$

The quantities with a superbar indicate those on the right hane side of the discontinuity. Above four equations hold for any y at $x=x$ within the enclosure. Scalar-multiplying these equations with eithe $\phi_{m}^{\prime}$ or $\phi_{m}^{\prime \prime}$ and making use of the orthogonality relation (36) and (37) we then obtain

$$
\mathbf{V}^{\prime \prime}=\mathbf{P}^{\prime \prime} \overline{\mathbf{V}}^{\prime \prime}
$$




$$
\begin{aligned}
\mathbf{V}^{\prime}+\mathbf{R}^{\prime \prime} \mathbf{V}^{\prime \prime} & =\mathbf{Q}^{\prime} \overline{\mathbf{V}}^{\prime}+\mathbf{S}^{\prime \prime} \overline{\mathbf{V}}^{\prime \prime} \\
\mathbf{I}^{\prime} & =\mathbf{P}^{\prime} \overline{\mathbf{I}}^{\prime} \\
\mathbf{R}^{\prime} \mathbf{I}^{\prime}+\mathbf{I}^{\prime \prime} & =\mathbf{S}^{\prime} \overline{\mathbf{I}}^{\prime}+\mathbf{Q}^{\prime \prime} \overline{\mathbf{I}}^{\prime \prime}
\end{aligned}
$$

where $\mathbf{V}^{\prime}$ and $\mathbf{I}^{\prime}$ are column vectors with the transmission-line voltage and current of the n-th LSE mode, $V_{n}^{\prime}\left(x_{l}\right)$ and $I_{n}^{\prime}\left(x_{l}\right)$, at the n-th position; similar definitions hold for $\mathbf{V}^{\prime \prime}$ and $\mathbf{I}^{\prime \prime}$ for the LSM modes and also for those vectors with a superbar. The $\mathbf{P}^{\prime}, \mathbf{P}^{\prime \prime}, \mathbf{Q}^{\prime}, \mathbf{Q}^{\prime \prime}, \mathbf{R}^{\prime}$, $\mathbf{R}^{\prime \prime}, \mathbf{S}^{\prime}$ and $\mathbf{S}^{\prime \prime}$, are matrices characterizing the coupling of the modes at the step discontinuity, and their elements are defined by scalar products or overlap integrals of the mode functions on the two sides of the discontinuity as follows:

$$
\begin{aligned}
P_{m n}^{\prime} & =Q_{m n}^{\prime}=<\phi_{m}^{\prime} \mid \bar{\phi}_{n}^{\prime}> \\
P_{m n}^{\prime \prime} & =<\phi_{m}^{\prime \prime}\left|\frac{1}{\bar{\epsilon}(y)}\right| \bar{\phi}_{n}^{\prime \prime}> \\
Q_{m n}^{\prime \prime} & =<\phi_{m}^{\prime \prime}\left|\frac{1}{\epsilon(y)}\right| \bar{\phi}_{n}^{\prime \prime}> \\
R_{m n}^{\prime} & =<\phi_{m}^{\prime \prime}\left|\frac{1}{\epsilon(y)}\right| \psi_{n}^{\prime}> \\
R_{m n}^{\prime \prime} & =<\phi_{m}^{\prime}\left|\psi_{n}^{\prime \prime}\right\rangle \\
S_{m n}^{\prime} & =<\phi_{m}^{\prime \prime}\left|\frac{1}{\epsilon(y)}\right| \bar{\psi}_{n}^{\prime}> \\
S_{m n}^{\prime \prime} & =<\phi_{m}^{\prime}\left|\bar{\psi}_{n}^{\prime \prime}\right\rangle
\end{aligned}
$$

for $m, n=1,2,3, \cdots$. It is evident from above equations that the matrices $\mathbf{P}^{\prime}{ }_{S}$ and $\mathbf{Q}^{\prime}$ s are responsible for the coupling among modes of the same polarization, whereas $\mathbf{R}^{\prime}$ s and $\mathbf{S}^{\prime}$ s are responsible for the cross-coupling among modes of opposite polarization.

It is proved that the following matrix identities hold for the present case:

$$
\mathbf{P}_{t}^{\prime} \mathbf{Q}^{\prime}=\mathbf{Q}_{t}^{\prime} \mathbf{P}^{\prime}=\mathbf{1}
$$




$$
\begin{aligned}
\mathbf{P}_{t}^{\prime \prime} \mathbf{Q}^{\prime \prime} & =\mathbf{Q}_{t}^{\prime \prime} \mathbf{P}^{\prime \prime}=\mathbf{1} \\
\mathbf{R}^{\prime} & =-\mathbf{R}_{t}^{\prime \prime} \\
\mathbf{S}^{\prime} & =\mathbf{Q}^{\prime \prime} \overline{\mathbf{R}}^{\prime} \\
\mathbf{S}^{\prime \prime} & =\mathbf{Q}^{\prime} \overline{\mathbf{R}}^{\prime \prime}
\end{aligned}
$$

where $\mathbf{1}$ is the unit matrix and $t$ stands for the transpose.

Using (46)-(49) and the above matrix identities, the relationship between the voltage and current on both sides of the l-th step discontinuity can be expressed in terms of coupling matrices as

$$
\mathbf{V}=\mathbf{Q}_{l} \overline{\mathbf{V}}, \quad \mathbf{I}=\mathbf{P}_{l} \overline{\mathbf{I}}
$$

or

$$
\mathbf{P}_{l t} \mathbf{V}=\overline{\mathbf{V}}, \quad \mathbf{Q}_{l t} \mathbf{I}=\overline{\mathbf{I}}
$$

In the above equations we define a super colum voltage and current vectors $\mathbf{V}, \overline{\mathbf{V}}, \mathbf{I}$ and $\overline{\mathbf{I}}$, and a super coupling matrices of the l-th step discontinuity $\mathbf{Q}_{l}$ and $\mathbf{P}_{l}$ by

$$
\begin{array}{cc}
\mathbf{V}=\left[\begin{array}{c}
\mathbf{V}^{\prime} \\
\mathbf{V}^{\prime \prime}
\end{array}\right] ; \quad \overline{\mathbf{V}}=\left[\begin{array}{l}
\overline{\mathbf{V}}^{\prime \prime} \\
\overline{\mathbf{V}}^{\prime \prime}
\end{array}\right] \\
\mathbf{I}=\left[\begin{array}{c}
\mathbf{I}^{\prime} \\
\mathbf{I}^{\prime \prime}
\end{array}\right] ; & \overline{\mathbf{I}}=\left[\begin{array}{l}
\overline{\mathbf{I}}^{\prime \prime} \\
\overline{\mathbf{I}}^{\prime \prime}
\end{array}\right] \\
\mathbf{Q}_{l}=\left[\begin{array}{ccc}
\mathbf{Q}^{\prime} & \mathbf{S}^{\prime \prime}-\mathbf{R}^{\prime \prime} \mathbf{P}^{\prime \prime} \\
\mathbf{0} & \mathbf{P}^{\prime \prime}
\end{array}\right] ; & \mathbf{P}_{l}=\left[\begin{array}{cc}
\mathbf{P}^{\prime} & \mathbf{0} \\
\mathbf{S}^{\prime}-\mathbf{R}^{\prime} \mathbf{P}^{\prime} & \mathbf{Q}^{\prime \prime}
\end{array}\right]
\end{array}
$$

It is straightforward to prove that the matrices $\mathbf{P}_{l}$ and $\mathbf{Q}_{l}$ satisfy the following identity

$$
\mathbf{P}_{l t} \mathbf{Q}_{l}=\mathbf{Q}_{l t} \mathbf{P}_{l}=\mathbf{1}
$$


If the input admittance matrix at $x=x_{l}^{+}$plane looking to the right is known as $\overrightarrow{\mathbf{Y}}\left(x_{l}^{+}\right)$, according to (50) and (51), it can be derived that the input admittance matrix $\overrightarrow{\mathbf{Y}}\left(x_{l}^{-}\right)$at $x=x_{l}^{-}$plane looking to the right satisfies

$$
\overrightarrow{\mathbf{Y}}\left(x_{l}^{-}\right)=\mathbf{P}_{l} \overrightarrow{\mathbf{Y}}\left(x_{l}^{+}\right) \mathbf{P}_{l t}
$$

where

$$
\mathbf{I}=\overrightarrow{\mathbf{Y}}\left(x_{l}^{+}\right) \mathbf{V} ; \quad \overline{\mathbf{I}}=\overrightarrow{\mathbf{Y}}\left(x_{l}^{-}\right) \overline{\mathbf{V}}
$$

On the other hand, if the input admittance matrix at $x=x_{\vec{l}}^{-}$plane looking to the left, $\overline{\mathbf{Y}}\left(x_{l}^{-}\right)$is known, the input admittance matrix at $x=x_{l}^{-}$plane looking to the left $\overline{\mathbf{Y}}\left(x_{l}^{+}\right)$can be obtained as:

$$
\overline{\mathbf{Y}}\left(x_{l}^{+}\right)=\mathbf{Q}_{l t} \overline{\mathbf{Y}}\left(x_{l}^{-}\right) \mathbf{Q}_{l},
$$

The current reflection coefficient matrix $\Gamma\left(x_{l}^{-}\right)$at the $x=x_{l}^{-}$plane looking to the right can be obtained as

$$
\Gamma\left(x_{l}^{-}\right)=\left[\overrightarrow{\mathbf{Y}}\left(x_{l}^{-}\right)+\mathbf{Y}_{o l}\right]^{-1}\left[\overrightarrow{\mathbf{Y}}\left(x_{l}^{-}\right)-\mathbf{Y}_{o l}\right],
$$

and the input admittance matrix at the $x=x_{l-1}^{+}$plane looking to the right is determined by admittance transform technique as

$$
\overrightarrow{\mathbf{Y}}\left(x_{l-1}^{+}\right)=\mathbf{Y}_{o l}\left[\mathbf{I}+\mathbf{H}_{l} \boldsymbol{\Gamma}_{l} \mathbf{H}_{l}\right]\left[\mathbf{I}-\mathbf{H}_{l} \boldsymbol{\Gamma}_{l} \mathbf{H}_{l}\right]^{-1}
$$

where $\mathbf{Y}_{o l}$ and $\mathbf{H}_{l}$ are respectively, the characteristic admittance and phase matrices of the 1-th step discontinuity. They are all diagonal matrices, and their elements are 


$$
\begin{aligned}
\mathbf{H}_{\mathbf{l}} & =\left[\begin{array}{cc}
\mathbf{H}_{\mathbf{1}}^{\prime} & \mathbf{0} \\
\mathbf{0} & \mathbf{H}_{\mathbf{l}}^{\prime \prime}
\end{array}\right], \\
\mathbf{Y}_{\mathbf{o l}} & =\left[\begin{array}{cc}
\mathbf{Y}_{\mathrm{ol}}^{\prime} & \mathbf{0} \\
\mathbf{0} & \mathbf{Y}_{\mathrm{ol}}^{\prime \prime}
\end{array}\right] ; \\
\left(H_{l}^{\prime}\right)_{m n} & =\delta_{m n} \exp \left(-j k_{x l n}^{\prime} l_{l}\right), \\
\left(H_{l}^{\prime \prime}\right)_{m n} & =\delta_{m n} \exp \left(-j k_{x l n}^{\prime \prime} l_{l}\right), \\
\left(Y_{o l}^{\prime}\right)_{m n} & =\delta_{m n} Y_{o l n}^{\prime}, \\
\left(Y_{o l}^{\prime \prime}\right)_{m n} & =\delta_{m n} Y_{o l n}^{\prime \prime} .
\end{aligned}
$$

where $k_{x l n}$ and $Y_{o l n}$ are respectively, the wave number in the x direction and the characteristic admittance for the $\mathrm{n}$-th mode in the $\mathrm{l}$-th section.

Now let us come back to the whole discontinuity problem in the transverse cross section. Since the input admittance matrix at $x=x_{2}^{+}$ plane looking to the right is known as

$$
\overrightarrow{\mathbf{Y}}\left(x_{2}^{+}\right)=-j \mathbf{Y}_{03} \cot \left[k_{x 3}\left(x_{3}-x_{2}\right)\right] .
$$

By using (56), (59) and (60), the input admittance matrix at $x=x_{1}^{+}$ plane looking to the right, $\overrightarrow{\mathbf{Y}}\left(x_{1}^{+}\right)$can be obtained:

$$
\begin{aligned}
\overrightarrow{\mathbf{Y}}\left(x_{1}^{+}\right) & =\mathbf{Y}_{02}\left[\mathbf{I}+\mathbf{H}_{2} \boldsymbol{\Gamma}\left(x_{2}^{-}\right) \mathbf{H}_{2}\right]\left[\mathbf{I}-\mathbf{H}_{2} \boldsymbol{\Gamma}\left(x_{2}^{-}\right) \mathbf{H}_{2}\right]^{-1} \\
\Gamma\left(x_{2}^{-}\right) & =\left[\overrightarrow{\mathbf{Y}}\left(x_{2}^{-}\right)+\mathbf{Y}_{02}\right]^{-1}\left[\overrightarrow{\mathbf{Y}}\left(x_{2}^{-}\right)-\mathbf{Y}_{02}\right] \\
\overrightarrow{\mathbf{Y}}\left(x_{2}^{-}\right) & =\mathbf{P}_{2} \overrightarrow{\mathbf{Y}}\left(x_{2}^{+}\right) \mathbf{P}_{2 t} .
\end{aligned}
$$


Similarly, the input admittance matrix at $x=x_{1}^{+}$plane looking to the left, $\overrightarrow{\mathbf{Y}}\left(x_{1}^{+}\right)$can be obtained:

$$
\begin{aligned}
& \overline{\mathbf{Y}}\left(x_{1}^{+}\right)=\mathbf{Q}_{\mathbf{1}} \overline{\mathbf{Y}}\left(x_{1}^{-}\right) \mathbf{Q}_{\mathbf{1}} \\
& \overline{\mathbf{Y}}\left(x_{1}^{-}\right)=-j \mathbf{Y}_{01} \cot \left(k_{x \mathbf{1}} x_{1}\right)
\end{aligned}
$$

Refering to Fig.2, the relationship between the voltage and current at $x=x_{1}^{+}$plane may be expressed by:

$$
\begin{aligned}
& \mathbf{I}_{2}\left(x_{1}^{+}\right)=\overrightarrow{\mathbf{Y}}\left(x_{1}^{+}\right) \mathbf{V}_{2}\left(x_{1}^{+}\right) \\
& \mathbf{I}_{2}\left(x_{1}^{+}\right)=-\overline{\mathbf{Y}}\left(x_{1}^{+}\right) \mathbf{V}_{2}\left(x_{1}^{+}\right)
\end{aligned}
$$

where subscript 2 and later 1,3 indicates the different constituent regions. The negative sign in (68) reflects the fact that the current in the transmission line flows in the positive $\mathrm{x}$ direction, while the admittance $\overleftarrow{\mathbf{Y}}\left(x_{1}^{+}\right)$is defined with respect to the opposite direction. Evidently, (67) and (68) are two different equations relating the same set of voltages to the same set of currents. Equating (67) and (68), we obtain

$$
\mathbf{Y}\left(x_{1}^{+}\right) \mathbf{V}_{2}\left(x_{1}^{+}\right)=0
$$

where

$$
\mathbf{Y}\left(x_{1}^{+}\right)=\overrightarrow{\mathbf{Y}}\left(x_{1}^{+}\right)+\overline{\mathbf{Y}}\left(x_{1}^{+}\right)
$$

For the linear homogeneous system of equations (69) the necessary and sufficient condition for the existence of a nontrival solution is that the determinant of the coefficient matrix vanishes, namely, 


$$
\operatorname{det} \mathbf{Y}\left(x_{1}^{+}\right)=0 .
$$

In other words, this is a condition under which non-zero current may exist in the absence of any source excitation in the network of Fig.2. Since the admittances in (71) are all functions of the longitudinal propagation constant $k_{z}$, the eigenvalue of the sample filled waveguide; therefore, (71) is the equation to determine the allowable values of $k_{z i}$ for the $\mathrm{i}$-th mode, and is actually the dispersion equation for the sample filled waveguide. Once the eigenvalues are obtained, the voltage at $\mathrm{x}=x_{1}^{+}$plane can be determined by equation (69) in following way.

Let $\mathbf{V}_{2}\left(x_{1}^{+}\right)=\mathbf{f}$, and $\mathbf{f}=\left(f_{1}, f_{2}, \cdots\right)$, then from (69), we have

$$
\mathbf{Y}\left(x_{1}^{+}\right) \mathbf{f}=0 .
$$

Equation (72), though simple in form, involves matrices of infinite order which must be truncated to obtain numerical approximations to prescribed accuracy. If the truncation number is chosen to be $\mathrm{m}$, only m-1 solutions are independent for the linear homogeneous system of equations. We thus can rearrange equation (69) as follows

$$
\left\{\begin{array}{c}
Y_{22} f_{2}+Y_{23} f_{3}+\cdots+Y_{2 m} f_{m}=-Y_{21} f_{1} \\
Y_{32} f_{2}+Y_{33} f_{3}+\cdots+Y_{3 m} f_{m}=-Y_{31} f_{1} \\
\vdots \\
\vdots \\
Y_{m 2} f_{2}+Y_{m 3} f_{3}+\cdots+Y_{m m} f_{m}=-Y_{m 1} f_{1} .
\end{array}\right.
$$

This is a (m-1)-dimensional linear inhomogeneous system of equations. We may let $f_{1}=1$ temporarily so that the equations can be solved with some numerical methods, say, Gauss eliminate method, and the relationship between $f_{\alpha}(\alpha=2,3, \cdots, m)$ and $f_{1}$ is then known. 
In order to determine the eigenfunctions of the sample filled waveguide with $f_{\alpha}$, let us define the n-th element of the voltage and current vectors in the three constituent regions as:

$$
\begin{gathered}
\begin{cases}V_{1 n}=A_{1 n} \sin \left(k_{1 x n} x\right), & \left(0 \leq x \leq x_{1}\right) ; \\
V_{2 n}=A_{2 n} \sin \left(k_{2 x n} x+p_{2 n}\right), & \left(x_{1} \leq x \leq x_{2}\right) ; \\
V_{3 n}=A_{3 n} \sin \left(k_{3 x n}\left(x-x_{3}\right)\right), & \left(x_{2} \leq x \leq x_{3}\right)\end{cases} \\
\begin{cases}I_{1 n}=j Y_{1 n} A_{1 n} \cos \left(k_{1 x n} x\right), & \left(0 \leq x \leq x_{1}\right) \\
I_{2 n}=j Y_{2 n} A_{2 n} \cos \left(k_{2 x n} x+p_{2 n}\right), & \left(x_{1} \leq x \leq x_{2}\right) \\
I_{3 n}=j Y_{3 n} A_{3 n} \cos \left(k_{3 x n}\left(x-x_{3}\right)\right), & \left(x_{1} \leq x \leq x_{2}\right)\end{cases}
\end{gathered}
$$

The amplitudes and phases in (74) and (75) for each region can be obtained as follows:

In Region 1: $\left(0 \leq x \leq x_{1}\right)$

From

$$
\mathbf{V}_{\mathbf{1}}\left(x_{1}^{-}\right)=\mathbf{Q}_{\mathbf{1}} \mathbf{V}_{2}\left(x_{1}^{+}\right)=\mathbf{Q}_{1} \mathbf{f}=\mathbf{g}=\left(g_{1}, g_{2}, \cdots, g_{n}, \cdots, g_{m}\right)_{t},
$$

we obtain

$$
A_{1 n} \sin \left(k_{x 1 n} x_{1}\right)=g_{n},
$$

or

$$
A_{1 n}=g_{n} / \sin \left(k_{x 1 n} x_{1}\right)
$$

In Region 2: $\left(x_{1} \leq x \leq x_{2}\right)$

From

$\mathbf{I}_{2}\left(x_{1}^{+}\right)=\overrightarrow{\mathbf{Y}}\left(x_{1}^{+}\right) \mathbf{V}_{2}\left(x_{1}^{+}\right)=\overrightarrow{\mathbf{Y}}\left(x_{1}^{+}\right) \mathbf{f}=\mathbf{h}=\left(h_{1}, h_{2}, \cdots, h_{n}, \cdots, h_{m}\right)_{t}$,

$$
\mathbf{V}_{2}\left(x_{1}^{+}\right)=\mathbf{f}=\left(f_{1}, f_{2}, \cdots, f_{n}, \cdots, f_{m}\right)_{t}
$$


we have

$$
\begin{array}{r}
j Y_{2 n} A_{2 n} \cos \left(k_{x 2 n} x_{1}+p_{2 n}\right)=h_{n}, \\
A_{2 n} \sin \left(k_{x 2 n} x_{1}+p_{2 n}\right)=f_{n} ;
\end{array}
$$

or

$$
\begin{array}{r}
p_{2 n}=\arctan \left(j Y_{2 n} f_{n} / h_{n}\right)-k_{x 2 n} x_{1} ; \\
A_{2 n}=f_{n} / \sin \left(k_{x 2 n} x_{1}+p_{2 n}\right) .
\end{array}
$$

In Region 3: $\left(x_{2} \leq x \leq x_{3}\right)$

Since the amplitudes and phases of the voltage in Region 2 are determined, it means that each element of the voltage vector at $x=x_{2}^{-}$ plane is obtained as:

$$
V_{2 n}\left(x_{2}^{-}\right)=A_{2 n} \sin \left(k_{x 2 n} x_{2}+p_{2 n}\right)
$$

therefore, from

$$
\mathbf{V}_{3}\left(x_{2}^{+}\right)=\mathbf{P}_{2 t} \mathbf{V}_{2}\left(x_{2}^{-}\right)=\mathbf{d}=\left(d_{1}, d_{2}, \cdots, d_{n}, \cdots, d_{m}\right)_{t}
$$

we get

$$
A_{3 n} \sin \left(k_{x 3 n}\left(x_{2}-x_{3}\right)\right)=d_{n}
$$

or

$$
A_{3 n}=d_{n} / \sin \left(k_{x 3 n}\left(x_{2}-x_{3}\right)\right)
$$

Through the above procedure, all the amplitudes and phases of the voltages and currents in the three regions can be determined once f is obtained. As mentioned before, the elements $f_{i}(i=2,3, \cdot, m)$ of vector $\mathbf{f}$ can be expressed by $f_{1}$ which can be now determined by the orthonormal relation of the modefunctions in the sample filled waveguide as

$$
f_{1}=1 / \sqrt{A}
$$


where

$$
\begin{aligned}
& A=\iint_{S}(\mathbf{e} \times \mathbf{h}) \cdot \mathbf{d} \mathbf{s}=\sum_{\Delta}^{3} \iint_{\Delta}(\mathbf{e} \times \mathbf{h}) \cdot \mathbf{d} \mathbf{s}=\sum_{\Delta}^{3} A^{\Delta} \\
& A^{\Delta}=\iint_{\Delta} e_{x}^{\prime} h_{y}^{\prime} d x d y+\iint_{\Delta} e_{x}^{\prime \prime} h_{y}^{\prime} d x d y+\left(-\iint_{\Delta} e_{y}^{\prime \prime} h_{x}^{\prime} d x d y\right) \\
& +\left(-\iint_{\Delta} e_{y}^{\prime \prime} h_{x}^{\prime \prime} d x d y\right) \\
& =S_{1}+S_{2}+S_{3}+S_{4} \\
& S_{1}=\sum_{m}^{N L S E} \frac{\epsilon_{0}}{\mu_{0}} \frac{-\left(k_{u m}^{\prime}\right)^{2}}{\left(k_{x m}^{\prime}\right)^{4}} \int_{x_{a}}^{x_{b}}\left[\frac{d V_{m}^{\prime}(x)}{d x}\right]^{2} d x \\
& S_{2}=\sum_{m}^{N L S E} \sum_{n}^{N L S M} \frac{\epsilon_{0}}{\mu_{0}} \frac{\left(k_{u m}^{\prime}\right)^{2}}{\left(k_{x m}^{\prime}\right)^{2}\left(k_{u n}^{\prime \prime}\right)^{2} k_{z}}\left[\int_{x_{a}}^{x_{b}} \frac{d V_{m}^{\prime}(x)}{d x} \frac{d V_{n}^{\prime \prime}(x)}{d x} d x\right] \\
& \times\left[\int_{0}^{b} \phi_{m}^{\prime}(y) \frac{1}{\epsilon(y)} \frac{d \phi_{n}^{\prime \prime}(y)}{d y} d y\right] \\
& S_{3}=\sum_{m}^{N L S E} \sum_{n}^{N L S M} \frac{\epsilon_{0}}{\mu_{0}} \frac{1}{k_{z}}\left[\int_{x_{a}}^{x_{b}} V_{m}^{\prime}(x) V_{n}^{\prime \prime}(x) d x\right] \\
& \times\left[\int_{0}^{b} \phi_{n}^{\prime \prime}(y)-\frac{1}{\epsilon(y)} \frac{d \phi_{m}^{\prime}(y)}{d y} d y\right] \\
& S_{4}=\sum_{m}^{N L S M} \frac{\epsilon_{0}}{\mu_{0}} \frac{-k_{0}^{2}}{\left(k_{u m}^{\prime \prime}\right)^{2}} \int_{x_{a}}^{x_{b}}\left[V_{m}^{\prime}(x)\right]^{2} d x
\end{aligned}
$$




\section{STEP II. Calculation of the Scattering Characteristics of the Discontinuity in the Longitudinal Direction}

\section{Symmetrical consideration of the structure to facilitate the analysis procedure}

The 3-D discontinuity under consideration as shown in Fig.1 has two step discontinuities in the longitudinal cross section. However, the present structure is symmetrical in the longitudinal direction as shown in Fig.5. The scattering of a guided mode by such a symmetrical structure may be analyzed in terms of the symmetrical and anti-symmetrical excitations for which we have the open-circuit and short-circuit bisections respectively, as shown in Fig.5. It means that only one discontinuity is needed to be analyzed.

\section{Determination of the S-parameters for the discontinuity}

Referring to Fig.5, it is clear that the reflection coefficient for each guided mode at the symmetrical plane $\mathrm{z}=\mathrm{w} / 2\left(\mathrm{O}-\mathrm{O}^{\prime}\right.$ plane $)$ is 1.0 for the O.C. bisection or -1.0 for the S.C. bisection. Let $\mathbf{R}_{\mathrm{o}}$ and $\mathbf{R}_{\mathrm{s}}$ be the reflection coefficient matrices at $z=0^{-}$plane for the O.C. and the S.C. bisections respectively. The guided mode reflection coefficient matrix $\mathbf{R}$ (at $\mathrm{z}=0^{-}$plane) and the transmission matrix $\mathbf{T}$ (at $\mathrm{z}=\mathrm{W}$ plane) of the entire symmetrical structure are then given by

$$
\begin{aligned}
& \mathbf{R}=\left(\mathbf{R}_{\mathbf{o}}+\mathbf{R}_{\mathbf{s}}\right) / 2, \\
& \mathbf{T}=\left(\mathbf{R}_{\mathbf{o}}-\mathbf{R}_{\mathbf{s}}\right) / 2 .
\end{aligned}
$$

The scattering parameters $S_{11}=S_{22}$ and $S_{21}=S_{12}$ of the dominant mode in the empty waveguide are determined from the first row and the first column of $\mathrm{R}$ and $\mathrm{T}$ matrices respectively as

$$
\begin{aligned}
S_{11}=\mathbf{R}(1,1) & =\left[\mathbf{R}_{\mathbf{o}}(1,1)+\mathbf{R}_{\mathbf{s}}(1,1)\right] / 2, \\
S_{21} & =\mathbf{T}(1,1)=\left[\mathbf{R}_{\mathbf{o}}(1,1)-\mathbf{R}_{\mathbf{s}}(1,1)\right] / 2 ;
\end{aligned}
$$


where matrices $\mathbf{R}_{\mathrm{o}}$ and $\mathbf{R}_{\mathrm{s}}$ are determined by:

$$
\begin{aligned}
& \mathbf{R}_{\mathrm{o}}=\left[\mathbf{Z}_{\mathrm{o}}+\mathbf{Z}_{\mathrm{c}}\right]^{-1}\left[\mathbf{Z}_{\mathrm{o}}-\mathbf{Z}_{\mathrm{c}}\right] \\
& \mathbf{R}_{\mathbf{s}}=\left[\mathbf{Z}_{\mathrm{s}}+\mathbf{Z}_{\mathrm{c}}\right]^{-1}\left[\mathbf{Z}_{\mathrm{s}}-\mathbf{Z}_{\mathrm{c}}\right]
\end{aligned}
$$
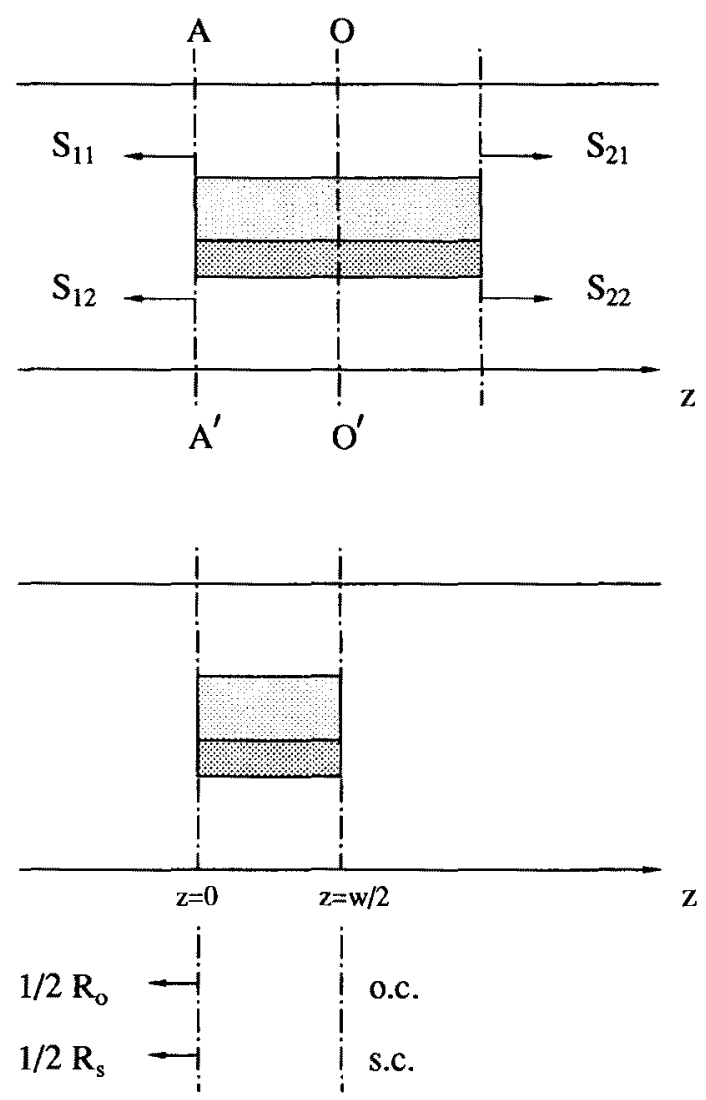

Fig.5 Symmetrical consideration and the equivalent network representation of the discontinuity in the longitudinal cross section. 
Here $\mathbf{Z}_{\mathrm{o}}$ and $\mathbf{Z}_{\mathrm{s}}$ are the input impedance matrices at $z=0^{-}$plane looking to the right for the open-circuit and short-circuit bisections of the $\mathrm{O}-\mathrm{O}^{\prime}$ plane respectively. They are determined by:

$$
\begin{aligned}
& \mathbf{Z}_{\mathbf{o}}=\mathbf{M} \overline{\mathbf{Z}}_{\mathbf{o}} \mathbf{M}_{\mathbf{t}}, \\
& \mathbf{Z}_{\mathbf{s}}=\mathbf{M} \overline{\mathbf{Z}}_{\mathbf{s}} \mathbf{M}_{\mathbf{t}}
\end{aligned}
$$

where $\overline{\mathbf{Z}}_{o}$ and $\overline{\mathbf{Z}}_{s}$ are input impedance matrices for the eigenmodes at $z=0^{+}$plane looking to the right for the open-circuit and shortcircuit bisections of the $\mathrm{O}-\mathrm{O}^{\prime}$ plane respectively. $\mathbf{Z}_{\mathrm{c}}$ is the characteristic impedance in the empty waveguide. $\overline{\mathbf{Z}}_{o}, \overline{\mathbf{Z}}_{s}$ and $\mathbf{Z}_{c}$ are all diagonal matrices, their elements are respectively given as follows

$$
\begin{aligned}
\mathbf{Z}_{\mathbf{c}} & =\left[\begin{array}{cc}
\mathbf{Z}_{\mathbf{c}}^{\prime} & \mathbf{0} \\
\mathbf{0} & \mathbf{Z}_{\mathbf{c}}^{\prime \prime}
\end{array}\right], \\
Z_{c n}^{\prime} & =\omega \mu_{0} / q_{z n}^{\prime}, \\
Z_{c n}^{\prime \prime} & =q_{z n}^{\prime \prime} / \omega \epsilon_{\mathbf{0}}, \\
\bar{Z}_{o n} & =-j \bar{Z}_{c n} \cot \left(\bar{k}_{z n} w / 2\right), \\
\bar{Z}_{s n} & =j \bar{Z}_{c n} \tan \left(\bar{k}_{z n} w / 2\right) ;
\end{aligned}
$$

where $\bar{Z}_{c n}$ is the characteristic impedance of the sample filled waveguide and is determined by (11) as

$$
\bar{Z}_{c n}=\bar{k}_{z n} / \omega \epsilon_{0}
$$

where $\mathbf{M}$ is the coupling matrix whose elements are determined in the next section. 


\section{Determination of the coupling matrices at the discontinuity plane}

At the discontinuity plane, or at $z=0$ cross section, the tangential components of the electromagnetic fields in the two waveguide must be continuous; from $(7)(8)$ and $(20)(21)$, in the $\Delta$ th region we have

$$
\begin{aligned}
\sum_{i} U_{i}^{\prime} \mathbf{e}_{\mathbf{i}}^{\prime}+\sum_{i} U_{i}^{\prime \prime} \mathbf{e}_{\mathbf{i}}^{\prime \prime} & =\sum_{j} \bar{U}_{j} \overline{\mathbf{e}}_{\mathbf{j}}, \\
\sum_{i} J_{i}^{\prime} \mathbf{h}_{\mathbf{i}}^{\prime}+\sum_{i} J_{i}^{\prime \prime} \mathbf{h}_{\mathbf{i}}^{\prime \prime} & =\sum_{j} \bar{J}_{j} \overline{\mathbf{h}}_{\mathbf{j}} .
\end{aligned}
$$

Here quantities with a superbar indicate the quantities in the sample filled waveguide. Cross multiplying $(89)$ by $\mathbf{h}_{\mathbf{i}}^{\prime}$ and $\mathbf{h}_{\mathbf{i}}^{\prime \prime}$ respectively from left; (90) by $\mathbf{e}_{\mathbf{i}}^{\prime}$ and $\mathbf{e}_{\mathbf{i}}^{\prime \prime}$ respectively from right, then integrating the resultant equations while making use of the orthogonal relation (5) and (6), we obtain

$$
\begin{aligned}
& U_{i}^{\prime}=\sum_{j} L_{i j} \bar{U}_{j}, \quad J_{i}^{\prime}=\sum_{j} A_{i j} \bar{J}_{j}, \\
& U_{i}^{\prime \prime}=\sum_{j} G_{i j} \bar{U}_{j}, \quad J_{i}^{\prime \prime}=\sum_{j} B_{i j} \bar{J}_{j}
\end{aligned}
$$

with

$$
\begin{aligned}
& L_{i, j}=\iint_{S}\left(\overline{\mathbf{e}}_{\mathbf{j}} \times \mathbf{h}_{\mathbf{i}}^{\prime}\right) \cdot \mathbf{d} \mathbf{s}, \\
& G_{i, j}=\iint_{S}\left(\overline{\mathbf{e}}_{\mathbf{j}} \times \mathbf{h}_{\mathbf{i}}^{\prime \prime}\right) \cdot \mathbf{d} \mathbf{s}, \\
& A_{i, j}=\iint_{S}\left(\mathbf{e}_{\mathbf{i}}^{\prime} \times \overline{\mathbf{h}}_{\mathbf{j}}\right) \cdot \mathbf{d} \mathbf{s}, \\
& B_{i, j}=\iint_{S}\left(\mathbf{e}_{\mathbf{i}}^{\prime \prime} \times \overline{\mathbf{h}}_{\mathbf{j}}\right) \cdot \mathbf{d} \mathbf{s} .
\end{aligned}
$$

Equations (91) and (92) may be written in matrix form as

$$
\begin{aligned}
\mathbf{U}^{\prime} & =\mathbf{L} \overline{\mathbf{U}}, \quad \mathbf{J}^{\prime}=\mathbf{A} \overline{\mathbf{J}} \\
\mathbf{U}^{\prime \prime} & =\mathbf{G} \overline{\mathbf{U}}, \quad \mathbf{J}^{\prime \prime}=\mathbf{B} \overline{\mathbf{J}}
\end{aligned}
$$


If we define a super column voltage vector $\mathbf{U}$ with the column vectors $\mathbf{U}^{\prime}$ and $\mathbf{U}^{\prime \prime}$ as its elements, and similarly for $\mathbf{J}$ as

$$
\mathbf{U}=\left(\begin{array}{c}
\mathbf{U}^{\prime} \\
\mathbf{U}^{\prime \prime}
\end{array}\right), \quad \mathbf{J}=\left(\begin{array}{c}
\mathbf{J}^{\prime} \\
\mathbf{J}^{\prime \prime}
\end{array}\right)
$$

we can obtain

$$
\mathbf{U}=\mathbf{M} \overline{\mathbf{U}}, \quad \mathbf{J}=\mathbf{N} \overline{\mathbf{J}}
$$

where

$$
\mathbf{M} \doteq\left(\begin{array}{l}
\mathbf{L} \\
\mathbf{G}
\end{array}\right), \quad \mathbf{N}=\left(\begin{array}{l}
\mathbf{A} \\
\mathbf{B}
\end{array}\right)
$$

It can be proved that

$$
\mathbf{M}_{t} \mathbf{N}=\mathbf{N}_{t} \mathbf{M}=\mathbf{1}
$$

which states that the inverses of the infinite matrices $\mathbf{M}$ and $\mathbf{N}$ may be simply obtained from the transposes of $\mathbf{N}$ and $\mathbf{M}$. Using above matrix identity, (99) may be rewritten as

$$
\mathbf{N}_{t} \mathbf{U}=\overline{\mathbf{U}}, \quad \mathbf{M}_{t} \mathbf{J}=\overline{\mathbf{J}}
$$

According to the definition of the impedance of the multimode network we have

$$
\mathbf{U}=\mathbf{Z J}, \quad \overline{\mathbf{U}}=\overline{\mathbf{Z J}}
$$

From (99)-(101), we get

$$
\mathbf{Z}=\mathbf{M} \overline{\mathbf{Z}} \mathbf{M}_{\mathbf{t}}
$$

Mathematically, (102) corresponds with the linear complex space transformation; physically, it can be understood as an impedance transform formula which was just used in (87) and (88) to determine 
the impedance matrices $\mathbf{Z}_{\mathbf{o}}$ and $\mathbf{Z}_{\mathbf{s}}$.

Referring to Table 1 and Table 2 , the elements of the coupling matrices in (93)-(96) can be determined by the following formulae

$$
\begin{aligned}
L_{i j} & =\sum_{\Delta}^{3} \iint_{\Delta}\left(\overline{\mathbf{e}}_{\mathbf{j}} \times \mathbf{h}_{\mathbf{i}}^{\prime}\right) \cdot \mathbf{i}_{\mathbf{z}} d x d y=\sum_{\Delta}^{3} L_{i j}^{\Delta} \\
L_{i j}^{\Delta} & =\iint_{\Delta} \bar{e}_{x j}^{\prime} h_{y j}^{\prime} d x d y+\iint_{\Delta} \bar{e}_{x j}^{\prime \prime} h_{y j}^{\prime} d x d y+\left(-\iint_{\Delta} \bar{e}_{y j}^{\prime \prime} h_{x i}^{\prime} d x d y\right) \\
& =S_{L 1}+S_{L 2}+S_{L 3}
\end{aligned}
$$$$
S_{L 1}=\sum_{m}^{N L S E} \sqrt{\frac{\epsilon_{0}}{\mu_{0}}} \frac{k_{0}^{2}}{\left(\bar{k}_{x m j}^{\prime}\right)^{2}} \frac{j A_{i}^{\prime} k_{y i}^{\prime}}{k_{0}}\left[\int_{x_{a}}^{x_{b}} \frac{d \bar{V}_{m j}^{\prime}}{d x} \cos k_{x i}^{\prime} x d x\right]
$$$$
\times\left[\int_{0}^{b} \bar{\phi}_{m}^{\prime}(y) \sin k_{y i}^{\prime} y d y\right]
$$$$
\begin{aligned}
S_{L 2}= & \sum_{m}^{N L S M} \sqrt{\frac{\epsilon_{0}}{\mu_{0}}} \frac{-k_{0}^{2}}{\left(\bar{k}_{u m}^{\prime \prime}\right)^{2}} \frac{j A_{i}^{\prime} k_{y i}^{\prime}}{k_{0} \bar{k}_{z j}}\left[\int_{x_{a}}^{x_{b}} \frac{d \bar{V}_{m j}^{\prime \prime}}{d x} \cos k_{x i}^{\prime} x d x\right] \\
& \times\left[\int_{0}^{b} \frac{{\overline{d \phi_{m}^{\prime \prime}}}^{\prime \prime}(y)}{d y} \frac{1}{\bar{\epsilon}(y)} \sin k_{y i}^{\prime} y d y\right]
\end{aligned}
$$$$
S_{L 3}=\sum_{m}^{N L S M} \sqrt{\frac{\epsilon_{0}}{\mu_{0}}} \frac{j k_{0} A_{i}^{\prime} k_{x i}^{\prime}}{\bar{k}_{z j}}\left[\int_{x_{a}}^{x_{b}} \bar{V}_{m j}^{\prime \prime} \sin k_{x i}^{\prime} x d x\right]
$$$$
\times\left[\int_{0}^{b} \bar{\phi}_{m}^{\prime \prime}(y) \frac{1}{\bar{\epsilon}(y)} \cos k_{y i}^{\prime} y d y\right]
$$ 


$$
\begin{aligned}
& G_{i j}=\sum_{\Delta}^{3} \iint_{\Delta}\left(\overline{\mathbf{e}}_{\mathbf{j}} \times \mathbf{h}_{\mathbf{i}}{ }^{\prime}\right) \cdot \mathbf{i}_{\mathbf{z}} d x d y=\sum_{\Delta}^{3} G_{i j}^{\Delta} \\
& G_{i j}^{\Delta}=\iint_{\Delta} \bar{e}_{x j}^{\prime} h_{y j}^{\prime \prime} d x d y+\iint_{\Delta} \bar{e}_{x j}^{\prime \prime} h_{y j}^{\prime \prime} d x d y+\left(-\iint_{\Delta} \bar{e}_{y j}^{\prime \prime} h_{x i}^{\prime \prime} d x d y\right) \\
& =S_{G 1}+S_{G 2}+S_{G 3} \\
& S_{G \mathbf{1}}=\sum_{m}^{N L S E} \sqrt{\frac{\epsilon_{0}}{\mu_{0}}} \frac{k_{0}^{2}}{\left(\bar{k}_{x m j}^{\prime}\right)^{2}} \frac{j A_{i}^{\prime \prime} k_{x i}^{\prime \prime}}{k_{0}}\left[\int_{x_{a}}^{x_{b}} \frac{d \bar{V}_{m j}^{\prime}}{d x} \cos k_{x i}^{\prime \prime} x d x\right] \\
& \times\left[\int_{0}^{b} \bar{\phi}_{m}^{\prime}(y) \sin k_{y i}^{\prime \prime} y d y\right] \\
& S_{G 2}=\sum_{m}^{N L S M} \sqrt{\frac{\epsilon_{0}}{\mu_{0}}} \frac{-k_{0}^{2}}{\left(\bar{k}_{u m}^{\prime \prime}\right)^{2}} \frac{j A_{i}^{\prime \prime} k_{x i}^{\prime \prime}}{k_{0} \bar{k}_{z j}}\left[\int_{x_{a}}^{x_{b}} \frac{d \bar{V}_{m j}^{\prime \prime}}{d x} \cos k_{x i}^{\prime \prime} x d x\right] \\
& \times\left[\int_{0}^{b} \frac{\overline{\phi \phi}_{m}^{\prime \prime}(y)}{d y} \frac{1}{\bar{\epsilon}(y)} \sin k_{y i}^{\prime \prime} y d y\right] \\
& S_{G 3}=\sum_{m}^{N L S M} \sqrt{\frac{\epsilon_{0}}{\mu_{0}}} \frac{-j k_{0} A_{i}^{\prime \prime} k_{y i}^{\prime \prime}}{\bar{k}_{z j}}\left[\int_{x_{a}}^{x_{b}} \bar{V}_{m j}^{\prime \prime} \sin k_{x i}^{\prime \prime} x d x\right] \\
& \times\left[\int_{0}^{b} \bar{\phi}_{m}^{\prime \prime}(y) \frac{1}{\bar{\epsilon}(y)} \cos k_{y i}^{\prime \prime} y d y\right]
\end{aligned}
$$




$$
\begin{aligned}
& A_{i j}=\sum_{\Delta}^{3} \iint_{\Delta}\left(\mathbf{e}_{\mathbf{i}}^{\prime} \times \overline{\mathbf{h}}_{\mathbf{j}}\right) \cdot \mathbf{i}_{\mathbf{z}} d x d y=\sum_{\Delta}^{3} A_{i j}^{\Delta} \\
& A_{i j}^{\Delta}=\iint_{\Delta} e_{x i}^{\prime} \bar{h}_{y j}^{\prime} d x d y+\left(-\iint_{\Delta} e_{y i}^{\prime} \bar{h}_{x j}^{\prime} d x d y\right)+\left(-\iint_{\Delta} e_{y i}^{\prime} \bar{h}_{x j}^{\prime \prime} d x d y\right) \\
& =S_{A 1}+S_{A 2}+S_{A 3} \\
& S_{A 1}=\sum_{m}^{N L S E} \sqrt{\frac{\epsilon_{0}}{\mu_{0}}} \frac{\left(\bar{k}_{u m}^{\prime}\right)^{2}}{\left(\bar{k}_{x m j}^{\prime}\right)^{2}} \frac{j A_{i}^{\prime} k_{y i}^{\prime}}{k_{0}}\left[\int_{x_{a}}^{x_{b}} \frac{d \bar{V}_{m j}^{\prime}}{d x} \cos k_{x i}^{\prime} x d x\right] \\
& \times\left[\int_{0}^{b} \bar{\phi}_{m}^{\prime}(y) \sin k_{y i}^{\prime} y d y\right] \\
& S_{A 2}=\sum_{m}^{N L S E} \sqrt{\frac{\epsilon_{0}}{\mu_{0}}} \frac{-j A_{i}^{\prime} k_{x i}^{\prime}}{k_{0}}\left[\int_{x_{a}}^{x_{b}} \bar{V}_{m j}^{\prime} \sin k_{x i}^{\prime} x d x\right] \\
& \times\left[\int_{0}^{b} \frac{d \bar{\phi}_{m}^{\prime}(y)}{d y} \cos k_{y i}^{\prime} y d y\right] \\
& S_{A 3}=\sum_{m}^{N L S M} \sqrt{\frac{\epsilon_{0}}{\mu_{0}}} \frac{k_{0}^{2}}{\left(\bar{k}_{u m}^{\prime \prime}\right)^{2}} \frac{j A_{i}^{\prime} k_{x i}^{\prime} \bar{k}_{z j}}{k_{0}}\left[\int_{x_{a}}^{x_{b}} \bar{V}_{m j}^{\prime \prime} \sin k_{x i}^{\prime} x d x\right] \\
& \times\left[\int_{0}^{b} \bar{\phi}_{m}^{\prime \prime}(y) \cos k_{y i}^{\prime} y d y\right]
\end{aligned}
$$




$$
\begin{aligned}
& B_{i j}=\sum_{\Delta}^{3} \iint_{\Delta}\left(\mathbf{e}_{\mathbf{i}}^{\prime \prime} \times \overline{\mathbf{h}}_{\mathbf{j}}\right) \cdot \mathbf{i}_{\mathbf{z}} d x d y=\sum_{\Delta}^{3} B_{i j}^{\Delta} \\
& B_{i j}^{\Delta}=\iint_{\Delta} e_{x i}^{\prime \prime} \bar{h}_{y j}^{\prime} d x d y+\left(-\iint_{\Delta} e_{y i}^{\prime \prime} \bar{h}_{x j}^{\prime} d x d y\right)+\left(-\iint_{\Delta} e_{y i}^{\prime \prime} \bar{h}_{x j}^{\prime \prime} d x d y\right) \\
& =S_{B 1}+S_{B 2}+S_{B 3} \\
& S_{B 1}=\sum_{m}^{N L S E} \sqrt{\frac{\epsilon_{0}}{\mu_{0}}} \frac{\left(\bar{k}_{u m}^{\prime}\right)^{2}}{\left(\bar{k}_{x m j}^{\prime}\right)^{2}} \frac{j A_{i}^{\prime \prime} k_{x i}^{\prime \prime}}{k_{0}}\left[\int_{x_{a}}^{x_{b}} \frac{d \bar{V}_{m j}^{\prime}}{d x} \cos k_{x i}^{\prime \prime} x d x\right] \\
& \times\left[\int_{0}^{b} \bar{\phi}_{m}^{\prime}(y) \sin k_{y i}^{\prime \prime} y d y\right] \\
& S_{B 2}=\sum_{m}^{N L S E} \sqrt{\frac{\epsilon_{0}}{\mu_{0}}} \frac{j A_{i}^{\prime \prime} k_{y i}^{\prime \prime}}{k_{0}}\left[\int_{x_{a}}^{x_{b}} \bar{V}_{m j}^{\prime} \sin k_{x i}^{\prime \prime} x d x\right] \\
& \times\left[\int_{0}^{b} \frac{{d \bar{\phi}_{m}^{\prime}}^{\prime}(y)}{d y} \cos k_{y i}^{\prime \prime} y d y\right] \\
& S_{B 3}=\sum_{m}^{N L S M} \sqrt{\frac{\epsilon_{0}}{\mu_{0}}} \frac{k_{0}^{2}}{\left(\bar{k}_{u m}^{\prime \prime}\right)^{2}} \frac{-j A_{i}^{\prime \prime} k_{y i}^{\prime \prime} \bar{k}_{z j}}{k_{0}}\left[\int_{x_{a}}^{x_{b}} \bar{V}_{m j}^{\prime \prime} \sin k_{x i}^{\prime \prime} x d x\right] \\
& \times\left[\int_{0}^{b} \bar{\phi}_{m}^{\prime \prime}(y) \cos k_{y i}^{\prime \prime} y d y\right]
\end{aligned}
$$




\section{NUMERICAL RESULTS}

In order to verify the reliability and efficiency of the present method, we have calculated the scattering characteristics of different II-VI semiconductor samples. Table 3 presents comparisons of the transmission characteristics for different samples with different gaps $g$ between the experimental data and the theoretical predictions. The input parameters for the samples are compiled in Table 4, while the data of the mounting structure is: $\varepsilon_{f}=2.3, \varepsilon_{a}=\varepsilon_{b}=\varepsilon_{w}=1.0$, $y_{4}-y_{3}=10.5 \mathrm{~mm}$ (cf. Fig.1). We did the measurements in a standard W/G 16 waveguide at a frequency of $8.89 \mathrm{GHz}$. It can be seen from the table that the agreement is very good for the transmission coefficient $S_{21}=\left|S_{21}\right| \exp \left(j \varphi_{21}\right)$. The reliability and the accuracy of the present method are thus justified.

Table 3. Comparisons of the transmission coefficients for different samples between the experimental data and the theoretical predictions

\begin{tabular}{|l|c|c|c|c|c|c|}
\hline \multirow{2}{*}{ Sample } & \multirow{2}{*}{$\mathrm{g}(\mathrm{mm})$} & $\mathrm{h}(\mathrm{mm})$ & \multicolumn{2}{|c|}{$\left|S_{21}\right|(\mathrm{dB})$} & \multicolumn{2}{c|}{$\varphi_{21}$ (degree) } \\
\cline { 4 - 7 } & & & exp. & theory & exp. & theory \\
\hline \hline Q114 & 1.26 & 0.2 & -10.58 & -10.68 & -82.59 & -87.04 \\
(CdHgTe) & & & & & & \\
Q319 & 4.65 & 0.2 & -1.98 & -2.21 & -96.88 & -99.40 \\
(HgSe) & & & -2.05 & -2.14 & -71.80 & -76.81 \\
$\begin{array}{l}\text { Q310 } \\
\text { (HgSe) }\end{array}$ & 4.76 & 0.2 & -2.77 & -3.12 & -98.70 & -108.71 \\
$\begin{array}{l}\text { Q411 } \\
\text { (HgSe) }\end{array}$ & 4.96 & 0.2 & -1.79 & -1.79 & -62.51 & -60.51 \\
$\begin{array}{l}\text { Q114 } \\
\text { (CdHgTe) }\end{array}$ & 5.16 & 0.0 & -16 & & & \\
\hline
\end{tabular}


Table 4. Values of the sample parameters: dielectic constant and thickness of epitaxial layer and substrate, length of the sample. (cf. Fig.1)

\begin{tabular}{|c|c|c|c|r|r|}
\hline Sample & $\varepsilon_{p}$ & $\begin{array}{c}d_{p} \\
(\mu \mathrm{m})\end{array}$ & \multicolumn{1}{c|}{$\varepsilon_{s}$} & $\begin{array}{c}d_{s} \\
(\mathrm{~mm})\end{array}$ & $\begin{array}{c}w \\
(\mathrm{~mm})\end{array}$ \\
\hline \hline Q114 & $-704-j 1.276 \cdot 10^{5}$ & 4.7 & $11.0-j 0.5$ & 1.0 & 5.0 \\
Q310 & $-768-j 1.390 \cdot 10^{5}$ & 0.63 & $11.0-j 0.0$ & 0.5 & 6.6 \\
Q319 & $-893-j 1.612 \cdot 10^{5}$ & 1.1 & $11.0-j 0.0$ & 0.5 & 9.6 \\
Q411 & $-893-j 1.612 \cdot 10^{5}$ & 0.62 & $11.0-j 4.0$ & 0.8 & 10.0 \\
\hline
\end{tabular}

\section{ACKNOWLEDGEMENTS}

This work was supported by The National Natural Science Foundation of China and The Deutsche Forschungsgemeinschaft.

\section{REFERENCES}

[1] P. Greiner, L.Polignone, C.R. Becker, R. Geick, Appl. Phys. A55, 279 (1992).

[2] Xu Shanjia, Wu Xinzhang, P. Greiner C.R. Becker, R. Geick, Int'l. J. of Infrared and Millimeter Waves 13, 569 (1992).

[3] Xu Shanjia, Sheng Xinqing, P. Greiner, C.R. Becker, R. Geick, J. of Infrared and Millimeter Waves, 11, (1992).

[4] Xu Shanjia, Journal of Electronics 6, 50 (1989).

[5] Xu Shanjia, Journal of Electronics 6, 232 (1989).

[6] Xu Shanjia, S. T. Peng and F. K. Schwering, IEEE Trans. on MTT 37, 686 (1989). 